\title{
Advantages of Coherent Feedback for Cooling Quantum Oscillators
}

\author{
Ryan Hamerly* and Hideo Mabuchi ${ }^{\dagger}$
}

Edward L. Ginzton Laboratory, Stanford University, Stanford, California 94305, USA

(Received 31 May 2012; revised manuscript received 3 July 2012; published 23 October 2012)

\begin{abstract}
We model the cooling of open optical and optomechanical resonators via optical feedback in the linear quadratic Gaussian setting of stochastic control theory. We show that coherent feedback control schemes, in which the resonator is embedded in an interferometer to achieve all-optical feedback, can outperform the best possible linear quadratic Gaussian measurement-based schemes in the quantum regime of low steady-state excitation number. Such performance gains are attributed to the coherent controller's ability to process noncommuting output field quadratures simultaneously without loss of fidelity, and may provide important clues for the design of coherent feedback schemes for more general problems of nonlinear and robust control.
\end{abstract}

Feedback control of classical dynamical systems plays a central role in modern engineering [1] but its quantum analogue, the notion of controlling a quantum system via feedback with a quantum or classical controller, is much less developed. Recent progress in modeling [2-5] and realizing [6-15] quantum feedback underscores the need for systematic approaches to control design, as do the wide range of potential applications in quantum science and technology.

While some of the most interesting problems in quantum feedback control are nonlinear [16-21], linear open quantum systems provide a logical first step towards more general problems. Working with linear systems, James, Nurdin, and Petersen [22,23] have utilized interconnection models [24-26] based on quantum stochastic differential equations [27-29] to develop generalizations of the traditional $\mathcal{H}^{\infty}$ and linear quadratic Gaussian (LQG) control paradigms that allow for the possibility of coherent optical feedback with linear quantum controllers. Here we work within the quantum LQG framework of Ref. [23] to study steady-state cooling of open quantum oscillators such as optical and optomechanical resonators subject to stationary heating and damping, with optical probing and feedback. We utilize numerical optimization together with fundamental analytic results $[1,30]$ to describe stationary, autonomous coherent feedback control schemes [31] that outperform the best possible LQG measurement-based schemes. We find more systematic and quantitatively significant advantages of coherent feedback over measurement-based feedback than in linear control scenarios considered previously [12,23], and interpret these performance gains in terms of the way

Published by the American Physical Society under the terms of the Creative Commons Attribution 3.0 License. Further distribution of this work must maintain attribution to the author(s) and the published article's title, journal citation, and DOI. that noncommuting field quadratures propagate through the feedback loop.

We model quantum harmonic oscillators as cascadable open quantum systems using the SLH framework [32,33] and associated quantum stochastic differential equations. In the SLH framework, an open Markovian quantum system may be described as a triple $G=(S, L, H)$ where $S$ is a scattering matrix, $L$ is a coupling vector and $H$ is the system Hamiltonian. The SLH framework is a very general and powerful tool because it allows individual elements to be straightforwardly and systematically interconnected in a quantum circuit algebra [33]. For a linear system with a vector of internal state variables $x$, the quantum stochastic differential equations (analogues to the Heisenberg equations for open quantum systems $[27,34]$ ) take the following form $[22,23]$ :

$$
\begin{aligned}
d x(t) & =[A x(t)+a] d t+B d a(t) \\
d \tilde{a}(t) & =[C x(t)+c] d t+D d a(t) .
\end{aligned}
$$

Here $A, B, C, D, a$, and $c$ are real and related to $(S, L, H)$ in the Supplemental Material [35]; see also Refs. [22,36].

The processes $d a(t)$ and $d \tilde{a}(t)$ are Hermitian quantum stochastic processes for the inputs and outputs, respectively, defined by $d a_{i}=\left(d A_{i}+d A_{i}^{\dagger},\left(d A_{i}-d A_{i}^{\dagger}\right) / i\right)$, where $d A_{i}(t)=\int_{t}^{t+d t} a_{i}\left(t^{\prime}\right) d t^{\prime}$ is the quantum Wiener process [37,38] following the Itô rule $d A_{i} d A_{j}^{\dagger}=\delta_{i j} d t$ for vacuum inputs.

As a first example of a quantum control system, take a noisy optical cavity, Fig. 1 . This has the SLH model

$$
S=I, \quad L=\left[\sqrt{k_{1}} a, \sqrt{k_{2}} a, \sqrt{k_{3}} a\right], \quad H=\Delta a^{\dagger} a,
$$

where $\Delta$ is the detuning of the cavity resonance frequency from that of a rotating frame. The cavity is driven by two vacuum inputs (mirrors $k_{1}$ and $k_{2}$ ) and one thermal input (mirror $k_{3}$ ) and without feedback relaxes to a thermal state 


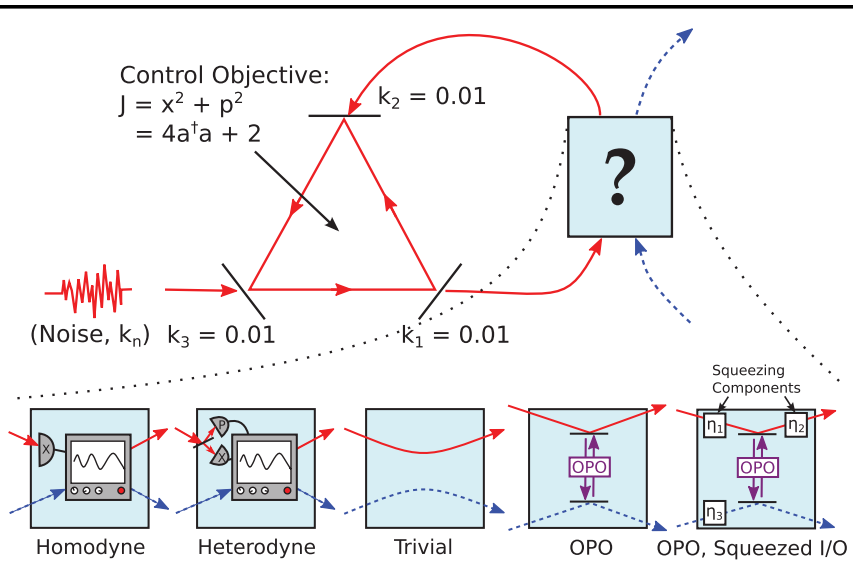

FIG. 1 (color). Possible classical and coherent cavity controllers for an optical cavity plant system.

with the mean photon number $\left\langle a^{\dagger} a\right\rangle$ proportional to the noise power $k_{n}$ [37]. The objective in this control problem is to minimize the effect of the noise on the cavity's internal state-in other words, to minimize the photon number $\left\langle a^{\dagger} a\right\rangle$ of the cavity. We accomplish this by sending output 1 through a control circuit and feeding the result back into input 2 . This is an LQG feedback control problem, assuming negligible control cost.

Once the full system is set up, with its associated $A, B$, $C$, and $D$ matrices, the covariance matrix $\sigma_{i j}=\frac{1}{2}\left\langle x_{i} x_{j}+\right.$ $\left.x_{j} x_{i}\right\rangle$ can be computed with the Lyapunov equation, $A \sigma+$ $\sigma A^{\mathrm{T}}+B F B^{\mathrm{T}}=0$, where $F$ is the input noise covariance; the mean photon number can then be computed from $\sigma$. Without a controller, we find:

$$
\left\langle a^{\dagger} a\right\rangle_{\mathrm{nc}}=\frac{k_{3}}{k_{1}+k_{2}+k_{3}} k_{n} .
$$

Five possible controllers are sketched in Fig. 1. The classical controllers (Homodyne and Heterodyne) work by measuring a quadrature from the cavity's output, or splitting the beam and measuring two different quadratures, and applying a feedback signal based on this measurement and the controller's internal state. The trivial controller works by feeding the output directly back into mirror 2 of the plant, perhaps with a phase shift. The remaining two coherent controllers have memory - the feedback signal depends not only on the probe field, but also on its history. The optical parametric oscillator (OPO) with squeezed input and output is the most general system realizable for two inputs and one internal degree of freedom [39].

The classical LQG optimization problem is convex, and the optimal controller is an analytic function of the plant parameters and the cost function [30]. The optimal homodyne and heterodyne controllers act as classical amplifiers. In the heterodyne case, the output $d \tilde{a}$ is related to the input $d a$ and vacuum noises $d a_{k 1}, d a_{k 2}$ by:

$$
\begin{aligned}
& d \tilde{a}_{x}=G\left(d a_{x}+d a_{k 1, x}\right)+d a_{k 2, x} \\
& d \tilde{a}_{p}=G\left(d a_{p}-d a_{k 1, p}\right)+d a_{k 2, p},
\end{aligned}
$$

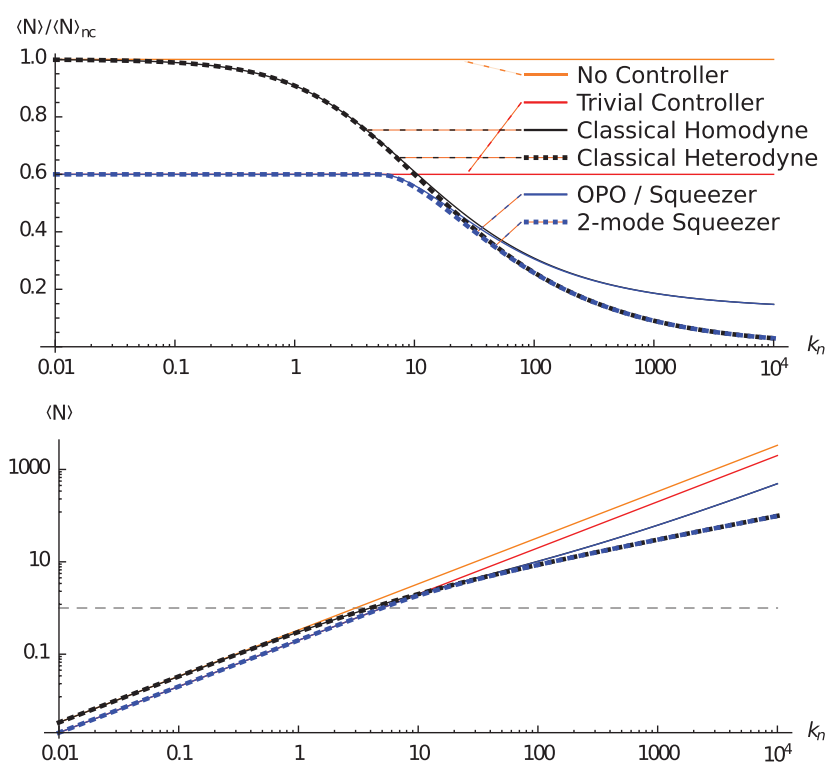

FIG. 2 (color). Bottom: Cavity photon number as a function of noise strength $k_{n}$, for various control schemes. Top: Photon number relative to the no-control case. Smaller is better.

where $G$ is the controller gain. The controller measures the probe, amplifies it, and then adds the signal to a vacuum channel. The plant mean photon number becomes

$$
\left\langle a^{\dagger} a\right\rangle_{\mathrm{cl}}=\frac{k_{2} \sinh ^{2} G+k_{3} k_{n}}{k_{1}+k_{2}+k_{3}+2 \sqrt{k_{1} k_{2}} \sinh G},
$$

which is plotted in dotted black in Fig. 2. Note that, while the controller does a good job in the classical regime, when $\left\langle a^{\dagger} a\right\rangle$ is high, it is ineffective in the quantum regime when the photon number is $\lesssim 1$.

Contrast this with the trivial controller, which feeds the output from mirror 1 directly into mirror 2. Rather than leaking photons separately, the two outputs then interfere constructively [18]. This leads to an enhancement in the dissipation, beating the best classical controller by a constant factor for small $k_{n}$.

$$
\left\langle a^{\dagger} a\right\rangle_{\mathrm{tr}}=\frac{k_{3}}{k_{1}+k_{2}+k_{3}+2 \sqrt{k_{1} k_{2}}} k_{n} .
$$

Numerical optimization of the most sophisticated controller we consider, the OPO with squeezed input and output, yields a locally optimal solution that can be shown to be equivalent [36] to a two-mode squeezer with squeezing $\eta$ :

$$
\begin{aligned}
& d \tilde{a}_{1}=\cosh (\eta) d a_{1}+\sinh (\eta) d a_{2} \\
& d \tilde{a}_{2}=\sinh (\eta) d a_{2}+\cosh (\eta) d a_{2} .
\end{aligned}
$$

This gives a mean photon number of:

$$
\left\langle a^{\dagger} a\right\rangle_{2 \text {-sq }}=\frac{k_{2} \sinh ^{2} \eta+k_{3} k_{n}}{k_{1}+k_{2}+k_{3}+2 \sqrt{k_{1} k_{2}} \cosh \eta},
$$


which is also plotted in Fig. 2. A single-OPO design can also be optimized, with results as shown in Fig. 2.

Notice just how similar Eqs. (5) and (8) are. Both the heterodyne controller and the linear amplifier reduce the cavity's photon number by amplifying the feedback signal, but also add noise to the system. For equivalent levels of amplification, the classical controller adds extra noise into the system from the measurement process. When $k_{n}$ and $\eta$ are large, this extra noise is negligible, but in the quantum regime where $k_{n}$ and $\eta$ are $\lesssim 1$, this noise can play a major role in making the linear amplifier outperform the heterodyne controller.

Of course, this need not be the global optimum. It is possible that other solutions, not found in our parameter search, lead to better LQG performance than the two-mode squeezer. But it is significant that we have found a quantum controller which does better than the optimal classical controller for all values of $k_{n}$, particularly in the quantum regime where the excitation number is low.

Our approach can also be applied to optomechanical oscillators (mechanical springs that couple to an optical field via a cavity), which have been a topic of tremendous recent interest in the physics community [40,41]. A central goal has been to find ways to exploit optomechanical coupling to cool the mechanical oscillator from ambient temperature to its ground state.

Consider a single cavity coupled to a mirror on a spring. If we shine a laser into the input port and adiabatically eliminate the internal mode, we obtain

$$
S=1_{2 \times 2}, \quad L=\left[K x_{m}, \sqrt{\Omega / Q} b\right], \quad H=\Omega b^{\dagger} b,
$$

where $K$ is a constant depending on the cavity and the drive field. It is not hard to show that this gives the following input-output relations [36]:

$$
\begin{aligned}
d x_{m} & =\Omega p_{m}-\Omega / 2 Q x_{m} \\
d p_{m} & =-\Omega x_{m}-\Omega / 2 Q p_{m}-2 K d a_{p} \\
d \tilde{a}_{x} & =d a_{x}+2 K x_{m} d t \quad d \tilde{a}_{p}=d a_{p} .
\end{aligned}
$$

The state variable $x_{m}$ is imprinted on the output $d \tilde{a}_{x}$, permitting the controller to be used as a measurement device via the $x$ quadrature. Conversely, the $p$-quadrature input $d a_{p}$ alters the state of the mirror, allowing the system to function as a feedback device.

Coupling two such cavities together as in Fig. 3, we obtain our model plant system. The cooling objective is to minimize the phonon number $\left\langle b^{\dagger} b\right\rangle$, where $b=\left(x_{m}+i p_{m}\right) / 2$ is the spring's annihilation operator. As in the optical cavity case, this is an LQG control problem.

Consider the first classical controller, the black line (labelled "classical") in the plot of Fig. 3. This controller only measures the $d a_{x}$ quadrature, since no information is contained in $d a_{p}$, and applies a feedback based on this
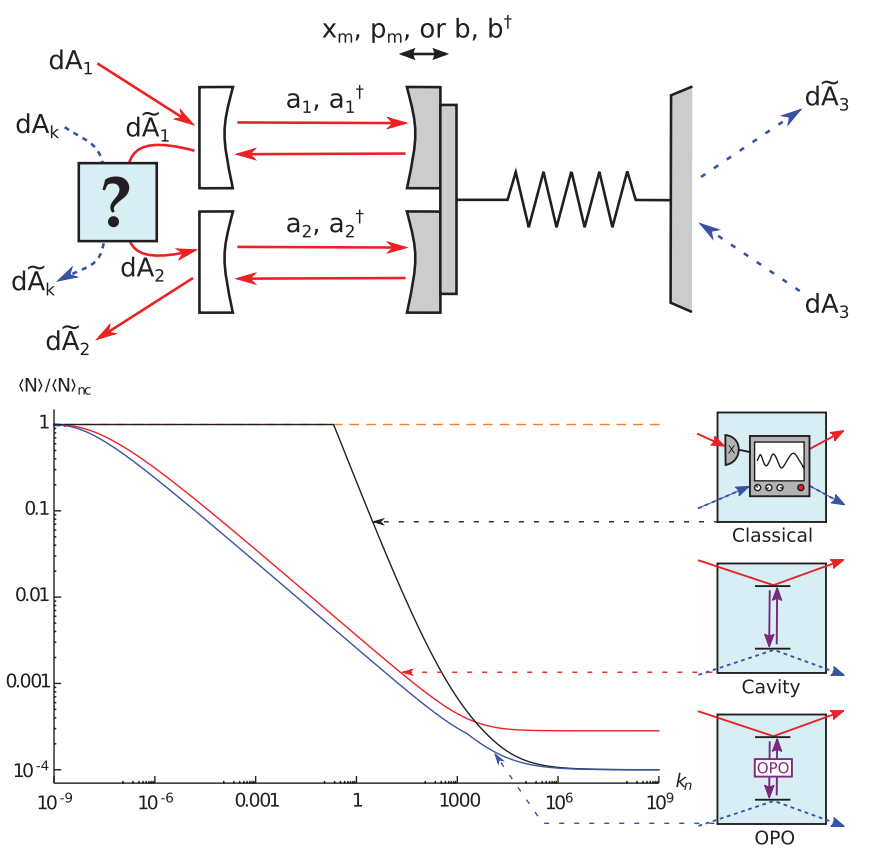

FIG. 3 (color). Control-system setup for the mechanical oscillator cooling problem with $\Omega=100, Q=10000$. Three potential controller designs and their relative performance.

input (such strategies outperform heterodyne-based classical control). When optimizing, we vary both the controller parameters and the couplings $K_{1}, K_{2}$ to the cavities in (9), since these depend on input laser powers rather than on properties of the plant. Here we will assume $K_{1}=-K_{2} \equiv$ $K$, however, as this type of design could be implemented practically using two modes of a single optical cavity with comparable power circulating in each. This symmetry in the control model also facilitates straightforward interpretation of the advantage of coherent feedback.

As before, the classical controller does a good job cooling the oscillator in the classical limit $\langle N\rangle \gg 1$, but does a very poor job in the quantum limit; below a threshold value, it has no effect at all. The classical controller adds two sources of noise to the plant. First, the probe field adds measurement noise to the spring through the first cavity. Second, the feedback field adds a feedback noise of equal magnitude via the second cavity. These noises add up incoherently. In the low- $k_{n}$ limit, they dominate the dynamics of the control system and the classical controller becomes ineffective.

We also optimized two types of coherent controller-an optical cavity and an OPO. They both behave qualitatively in a similar way, but the optical cavity is easier to understand. With the optical cavity controller, the feedback field is related to both the probe field and the cavity state, which changes with time:

$$
\begin{aligned}
d a & =(-i \Delta-\kappa / 2) a d t+\sqrt{\kappa} d \tilde{A}_{1} \\
d A_{2} & =d \tilde{A}_{1}+\sqrt{\kappa} a d t .
\end{aligned}
$$



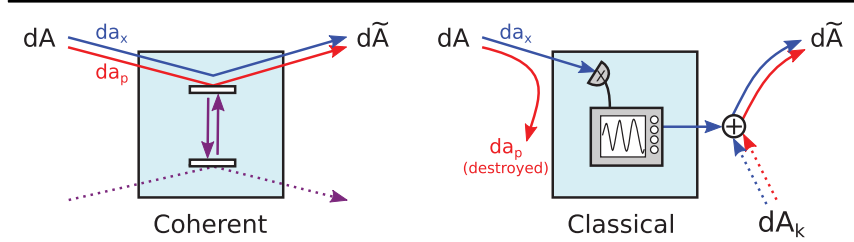

FIG. 4 (color). Flow of $x$ - and $p$-quadrature signals (blue and red, respectively) in the classical and coherent controllers.

Optimization shows that $\Delta=\Omega$, so both the cavity and mirror resonate at the same frequency. Although not interpreted in terms of feedback control theory, it is straightforward to show that cavity-optomechanical cooling experiments such as Refs. [9-11] have implemented an equivalent strategy in which the three optical cavities of Fig. 3 are collapsed into one. The LQG-optimal classical controller relies on a Kalman filter to estimate the plant state from the measured signal [1]. The coherent cavity controller can also be thought of as a Kalman filter, but one that preserves the coherence of the input signal $d \tilde{A}_{1}$. Classically the $p$-quadrature $d a_{p}$ is essentially discarded after the measurement, while in the cavity controller the field remains coherent and $d a_{p}$ coming out is the same as $d a_{p}$ going in. This correlates the noises in the measurement and feedback cavities such that the associated forces on the mechanical oscillator cancel, as shown in Fig. 4, giving the coherent cavity controller superior performance in the low phonon number regime. Previous studies had shown that optomechanical precision measurement schemes [42], particularly in the context of LIGO [43], may benefit from similar cancelations.

The OPO controller is more general than the cavity controller and its performance is slightly better over the whole noise range. As with the optical cavity case, it is significant that we have found realistic coherent control schemes that perform significantly better than provably optimal measurement-based schemes.

We have studied coherent control of linear quantum systems from an LQG perspective. In the systems studied, we have shown that there is always a quantum controller that does at least as well as the optimal classical controller. In the quantum regime, when the excitation number in the plant is of order unity, we have shown that the best quantum controller can do better-in some cases, significantly so. These results could be extended to nonquadratic cost functions in linear control systems. Indeed, some work has already been done on this matter, focusing on using coherent feedback to maximize the squeezing in a cavity mode [15]. Viewing an optomechanical device as a control system may also provide insight into minimizing the noise in optomechanical sensors. Finally, the fact that coherent controllers perform better because they utilize both quadratures of the input field may help guide the design of quantum controllers for nonlinear systems such as optical switches or memories.
This work has been supported by the NSF (PHY1005386), AFOSR (FA9550-11-1-0238) and DARPA-MTO (N66001-11-1-4106). Ryan Hamerly is supported by the NSF GRFP and a Stanford Graduate Fellowship. We thank Nikolas Tezak, Gopal Sarma, Dmitri Pavlichin, and Orion Crisafulli for useful discussions.

*rhamerly@stanford.edu

†hmabuchi@stanford.edu

[1] K. Åstrom and R. M. Murray, Feedback Systems: An Introduction for Scientists and Engineers (Princeton University Press, Princeton, NJ, 2008).

[2] V.P. Belavkin, Automation and Remote Control (English Translation) 44, 178 (1983).

[3] H. M. Wiseman and G. J. Milburn, Phys. Rev. Lett. 70, 548 (1993).

[4] AC. Doherty, S. Habib, K. Jacobs, H. Mabuchi, and S. M. Tan, Phys. Rev. A 62, 012105 (2000).

[5] H. M. Wiseman and G. J. Milburn, Quantum Measurement and Control (Springer, New York, 2009).

[6] W. P. Smith, J. E. Reiner, L. A. Orozco, S. Kuhr, and H. M. Wiseman, Phys. Rev. Lett. 89, 133601 (2002).

[7] M. A. Armen, J. K. Au, J. K. Stockton, A. C. Doherty, and H. Mabuchi, Phys. Rev. Lett. 89, 133602 (2002).

[8] P. Bushev, D. Rotter, A. Wilson, F. Dubin, C. Becher, J. Eschner, R. Blatt, V. Steixner, P. Rabl, and P. Zoller, Phys. Rev. Lett. 96, 043003 (2006).

[9] S. Gigan, H. R. Böhm, M. Paternostro, F. Blaser, G. Langer, J.B. Hertzberg, K. C. Schwab, D. Bäuerle, M. Aspelmeyer, and A. Zeilinger, Nature (London) 444, 67 (2006).

[10] O. Arcizet, P.-F. Cohadon, T. Briant, M. Pinard, and A. Heidmann, Nature (London) 444, 71 (2006).

[11] D. Kleckner and D. Bouwmeester, Nature (London) 444, 75 (2006).

[12] H. Mabuchi, Phys. Rev. A 78, 032323 (2008).

[13] G. G. Gillett, R. B. Dalton, B. P. Lanyon, M. P. Almeida, M. Barbieri, G. J. Pryde, J. L. O’Brien, K. J. Resch, S. D. Bartlett, and A. G. White, Phys. Rev. Lett. 104, 080503 (2010).

[14] C. Sayrin et al., Nature (London) 477, 73 (2011).

[15] S. Iida, M. Yukawa, H. Yonezawa, N. Yamamoto, and A. Furasawa, IEEE Trans. Autom. Control 57, 2045 (2012).

[16] J. Kerckhoff, H.I. Nurdin, D.S. Pavlichin, and H. Mabuchi, Phys. Rev. Lett. 105, 040502 (2010).

[17] J. Kerckhoff, D. S. Pavlichin, H. Chalabi, and H. Mabuchi, New J. Phys. 13, 055022 (2011).

[18] H. Mabuchi, Appl. Phys. Lett. 98, 193109 (2011).

[19] J. Zhang, R.-B. Wu, Y.-x. Liu, C.-W. Li, and T.-J. Tarn, IEEE Trans. Autom. Control 57, 1997 (2012).

[20] H. Amini et al., arXiv:1201.1387v1.

[21] M. Yanagisawa, Phys. Rev. Lett. 97, 190201 (2006).

[22] M. R. James, H. I. Nurdin, and I. R. Petersen, IEEE Trans. Autom. Control 53, 1787 (2008).

[23] H. I. Nurdin, M. R. James, and I. R. Petersen, Automatica 45, 1837 (2009).

[24] H. J. Carmichael, Phys. Rev. Lett. 70, 2273 (1993).

[25] C. W. Gardiner, Phys. Rev. Lett. 70, 2269 (1993). 
[26] M. Yanagisawa and H. Kimura, IEEE Trans. Autom. Control 48, 2107 (2003).

[27] R. L. Hudson and K. R. Parthasarathy, Commun. Math. Phys. 93, 301 (1984).

[28] A. Barchielli, in Open Quantum Systems III: Recent Developments, edited by S. Attal, A. Joye, and C.-A. Pillet (Springer, Berlin, 2006).

[29] C. W. Gardiner and M. J. Collett, Phys. Rev. A 31, 3761 (1985).

[30] D. Simon, Optimal State Estimation: Kalman $H^{\infty}$, and Nonlinear Approaches (John Wiley \& Sons, Hoboken, NJ, 2006).

[31] H. M. Wiseman and G. J. Milburn, Phys. Rev. A 49, 4110 (1994).

[32] J. Gough and M. R. James, Commun. Math. Phys. 287, 1109 (2009).

[33] J. Gough and M. R. James, IEEE Trans. Autom. Control 54, 2530 (2009).

[34] C. W. Gardiner, A. S. Parkins, and P. Zoller, Phys. Rev. A 46, 4363 (1992).
[35] See Supplemental Material at http://link.aps.org/ supplemental/10.1103/PhysRevLett.109.173602 for details on the SLH circuit algebra and its application to linear systems.

[36] R. Hamerly and H. Mabuchi, arXiv:1206.2688.

[37] C.W. Gardiner and P. Zoller, Quantum Noise: A Handbook of Markovian and Non-Markovian Quantum Stochastic Methods with Applications to Quantum Optics (Springer, New York, 2004).

[38] L. Bouten, R. Van Handel, and M. R. James, SIAM J. Control Optim. 46, 2199 (2007).

[39] H. I. Nurdin, M. R. James, and A.C. Doherty, SIAM J. Control Optim. 48, 2686 (2009).

[40] F. Marquardt and S. Girvin, Physics 2, 40 (2009).

[41] T. Botter, D. W. C. Brooks, N. Brahms, S. Schreppler, and D. M. Stamper-Kurn, Phys. Rev. A 85, 013812 (2012).

[42] M. Tsang and C. M. Caves, Phys. Rev. Lett. 105, 123601 (2010).

[43] O. Arcizet, T. Briant, A. Heidmann, and M. Pinard, Phys. Rev. A 73, 033819 (2006). 